\title{
Guidance is an intervention: experiences and reflections of message mediators and message recipients for 'alcohol in pregnancy' guidance.
}

Heather Trickey

Cardiff University

Rachel Brown ( $\sim$ brownr14@cardiff.ac.uk)

Cardiff University https://orcid.org/0000-0002-4475-1733

Research article

Keywords:

Posted Date: April 24th, 2020

DOI: https://doi.org/10.21203/rs.3.rs-24248/v1

License: (c) (i) This work is licensed under a Creative Commons Attribution 4.0 International License. Read Full License 


\section{Abstract}

Background

Heavy alcohol use in pregnancy is associated with poorer outcomes for babies, while evidence of harm from drinking at low levels is limited. UK guidance has been progressively strengthened, so that current guidance, underpinned by a precautionary principle, is to abstain from drinking alcohol if pregnant or planning a pregnancy. The aim of this study was to explore recipients' and mediators' awareness, experiences and reflections on the 2016 alcohol in pregnancy guidance.

Methods

Four focus groups explored the perspectives of policy makers, midwives, third sector advocates and new mothers. Transcripts were analysed thematically.

Results

Participants inferred a variety of lay programme theories to make sense of the precautionary principle, some of which may run counter to the intention of guidance developers. Participants also varied in their understanding of the role of message mediators, understandings included eliciting disclosures, communicating complexity, providing reassurance and contributing to social surveillance. Coherence of the guidance was challenged by paucity of underpinning evidence base, incongruence with the realities of pregnancy planning and a wider cultural norm of social drinking.

\section{Conclusions}

Improvements in efficacy may be achieved through recognition of guidance as a form of intervention in a complex system, underpinned by a logic model and with intended outcomes fully specified. Practical steps could be taken to nuance and layer communication of the underpinning evidence base. Communication strategies should carefully consider implications of 'scope creep' into pregnancy planning. Guidance could do more to address the role of partners and family members in supporting women's decisions.

\section{Background}

Many UK women consume at least some alcohol in pregnancy, with estimates varying from $40 \%^{1}$ to $75 \%^{2}$. A third report binge drinking - defined as six or more standard units per drinking occasion during early pregnancy, dropping to around $1 \%$ in the second trimester ${ }^{2}$. Rates of abstinence in pregnancy have been rising and, beyond the first trimester, most women (93\%) either do not consume alcohol or consume at levels below 1 units a week ${ }^{1}$. Less than $3 \%$ report heavier drinking (over 1-2 units per week) beyond the first trimester ${ }^{3}$.

Heavy alcohol use in pregnancy is associated with negative impacts including low birthweight, premature birth, miscarriage and foetal alcohol syndrome, with a clear dose-response relationship for level of alcohol use and risk of harm ${ }^{4}$. However, evidence of harm at low levels of consumption is limited $^{5}$.

\section{Guidance progression}

Over the past twenty years, UK public health guidance on drinking in pregnancy has been progressively refined. In the mid-1990 s, Department of Health advised pregnant women to drink no more than 1-2 units of alcohol per week ${ }^{6}$, based on evidence of harm at high levels of consumption and absence of harm at low levels. This was revised in 2007 to state that women who are pregnant, or trying to conceive, should abstain from alcohol completely, despite a commissioned evidence review supporting absence of evidence of harm associated with low levels of drinking ${ }^{7}$. The 2007 guidance also stated that if women chose to drink, they should consume no more than 1-2 units a week ${ }^{8}$. In January 2016, the Chief Medical Officers (CMO's) of the UK published new joint public health guidance on alcohol consumption for the general population, revising down recommended consumption for men. This document strengthened advice to women who are pregnant or planning a pregnancy, promoting compete abstinence and removing advice for women who chose to continue drinking 5 . 


\section{The precautionary principle}

The 2016 guidance document suggests that different underpinning principles were applied to the development of general population recommendations compared to those for women in pregnancy. Guidance for the general population is explicitly underpinned by a principle of 'informed choice'(p. 1) based on evidence of harm, while for pregnant women it is informed by a 'precautionary' approach (p. 8) in light of 'elusive ${ }^{9}$ evidence of harm from drinking in pregnancy at low levels. The rationale for adoption of a precautionary principle for pregnant women is absence of evidence of the safety of drinking rather than evidence of harm (p. 8).

The aim of this qualitative study was to explore message recipients' and mediators' awareness, experiences, and reflections on the content of the 2016 alcohol in pregnancy guidance and the use of the precautionary principle.

\section{Methods}

Four focus groups were convened to explore the perspectives of eighteen stakeholders on guidance for alcohol in pregnancy. The groups comprised:

1. Policy participants - two public health policy leads involved in alcohol policy in Wales and two third-sector policy professionals from charities concerned with preventing alcohol harm. All participants were known to the researchers through previous academic work.

2. Midwives - five midwives from three Local Health Boards (LHB's) across Wales, all with a remit for communicating alcohol advice in pregnancy. Two of these were specialists in supporting substance misuse issues. Participants had no prior knowledge of researchers but were recruited with the help of gatekeepers know to HT through previous research.

3. Third-sector advocates/information providers - four professionals from the NCT charity who are involved in utilising and communicating public health information to parents, face-to-face, by telephone and via a parent support helpline. Participants had no prior knowledge of researchers but were recruited with the help of gatekeepers know to HT through previous research.

4. New mothers - five mothers with babies under one year, recruited from an NCT postnatal group they attended. Participants had no prior knowledge of researchers.

Both researchers have significant experience in conducting qualitative research in the fields of alcohol research and infant/maternal health. Participants were first contacted by email, including a study information sheet and asking for expressions of interest. All participants were informed of the reason for the study and a brief professional background of the researchers, with the opportunity to ask further questions. All policy, health professional and third sector participants approached agreed to contribute to the study. Group 1 was conducted with two participants in attendance and two on conference call in the room at the host University, while groups 2-4 were conducted face to face (group 2 at the host university and groups 3 and 4 at an NCT setting). Group 4 (mothers) brought their babies with them. All groups were run by both female authors in their current research roles and no external parties were in attendance. Groups took 1-2 hours.

In preparation for the focus groups, the authors undertook an initial document review of historical and current guidance on alcohol and pregnancy, as well as the content and underpinning evidence for the UK CMO 2016 alcohol guidelines. This formed the basis of the topic guides developed by the authors and used throughout (Appendix 1). Due to time constraints, the topic guide was not pretested. Groups began with a statement-sorting task, based on key issues and controversies identified in this review (See Fig. 1), with participants organising statements by 'agree', 'disagree', 'not sure'. This was followed by a 5-minute presentation of key findings from the document review. A semi-structured topic guide was developed for the rest of the discussion, starting with discussion of the content of the sorting activity. Further discussion then included awareness and experience of the CMO's guidance; own experience of alcohol in pregnancy and awareness of the underpinning evidence (Appendix 1). After each group, the researchers discussed events and made any additional notes of relevance.

Recordings were transcribed in full and a coding frame developed through a two-phased, inductive and deductive approach, involving open reading for initial familiarisation, followed by identification of codes from both this and the document review. All transcripts were analysed thematically ${ }^{10}$ by each author, with ongoing discussion of divergence and contradiction in coding leading to final theme identification. Due to study time constraints, transcripts were not returned to participants for comment. 


\section{Results}

This section sets out participants' approach to sense-making - or lay theorising - about the principles underpinning the abstinence message and the roles of mediators in message communication. Three areas of challenge to the perceived coherence of the guidance are then presented: the paucity of evidence relating to drinking alcohol at low levels; lack of congruence between guidance to abstain and a spectrum of pregnancy planning behaviours; and wider social constraints on individual-level decision-making.

Interpretations of the precautionary principle

Participants interpreted the rationale for a precautionary principle and an abstinence message in three main ways. These were (i) to provide an extra layer of protection of the foetus, (ii) to reduce accidental immoderate drinking, (iii) to instigate long-term behaviour change within families.

\section{Protection for the foetus}

Participants across all groups suggested that a precautionary approach was intended to provide additional protection for unborn babies against the theoretical chance that alcohol might do them harm. Some felt that this was justified and was likely to be consistent with the personal agendas of most expectant mothers, who - it was assumed - would prefer to err on the side of caution to protect their baby. However, several participants in midwifery and third sector groups expressed concern that prioritising the theoretical harms to the foetus represented public health overreach into maternal autonomy, with an 'extra protection' rationale problematising the legal status of the foetus in relation to the mother:

It's the protection of the unborn child, isn't it? Which is a dichotomy, because it hasn't got any rights within the law, and yet we're still using the mother to enforce the [foetus's] rights.

\section{Group 2 - Midwife}

\section{Reducing accidental immoderate drinking}

Participants across all groups tended to think that the advice to abstain had been developed to reduce potential for misunderstandings that might arise from not knowing how much is 'too much', risking excessive and damaging consumption. Midwives most frequently suggested that their clients would prefer clear and straightforward messages over more complex content. Both midwives and mothers thought that women often underestimated the amount that they drank; some midwives felt that women were unable to count units, others had experience of women 'saving up'an allowance of alcohol to use on a planned drinking binge. Participants in the midwifery and mothers groups tended to believe that the clear and simple approach would help to safeguard the babies of more 'vulnerable' women who, they perceived, would struggle with a complex message - in particular, women with less education, drug or mental health issues:

I think for the clientele that I work with, I think having a clear, 'You should do this, you shouldn't do that', women know where they stand. And I think a lot of women get confused by so many different messages that sometimes it's easier to say, 'Right, You don't drink, or you shouldn't smoke...'

Group 2 - Midwife

By contrast, several participants in the policy, third sector and mothers groups, suggested that the lack of nuance was patronising: If you assume that pregnant women are somehow less capable of understanding complex messages, compared to the rest of the population, then there's a very good argument [for taking a precautionary approach].

Group 1 - Alcohol policy participant

\section{Changing family drinking behaviours}

Some in the policy and midwifery groups thought the precautionary guidance might be intended to have a longer-term impact on health behaviour, taking advantage of an intervention window during which women are particularly susceptible to health messages. Some believed that women's abstinence during pregnancy might lead to sustained benefits for families, perhaps stimulating 
permanent changes in consumption. However, this contradicted the experience of some mothers, who suggested that abstinence in pregnancy was made tolerable through knowing it was temporary.

Perceptions of the role of message mediators

Participants held contrasting understandings about the intended role of message mediators - health professionals, other expert advisors, family and friends and the general public - in communicating the abstinence message. This understandings included: (i) eliciting disclosures, thereby identifying women whose foetus is at risk and providing additional information or support, (ii) selectively communicating a more complex message, (iii) providing reassurance for women who had drunk alcohol before knowing they were pregnant and advising these women to stop drinking as soon as possible, (iv) re-enforcing social shaming and surveillance to prevent alcohol consumption from taking place.

\section{Eliciting disclosures from reluctant women}

Midwife participants felt that the clear abstinence message could act as a tool to elicit disclosures to help them to identify and support women who might have be inclined to underplay their drinking levels in antenatal appointments:

They might say, 'I have one or two units a week', they'll just disclose what they think they should say.

Group 2 - Midwife

In fact, midwives were far more prone to use the word 'disclosure' compared to other group participants and frequently as mothers being persuaded to talk when they may have been resistant. This contrasted with other uses, including an observation in the parent group that 'disclose' is potentially stigmatising. It was suggested in the policy group pressure to disclose might result in an unwanted referral to support services, leading to self-censorship among women, resulting in fewer disclosures.

\section{Communicating complexity}

Some midwife and third sector participants saw their role as nuancing and moderating the message, making it appropriate to the level of understanding of individuals they were addressing. Third sector participants understood their role as supporting informed choice, allowing expectant mothers to consider the complexity of the evidence. They agreed with midwives that different parents wanted different degrees of complexity, and sought ways to meet these varying needs:

I do feel like for some parents you just want to be told, 'Do I do this, or do I do that?' [...] for others, they do want to know everything, so, if they've made this decision, they want to know all the evidence behind it. All the reasoning.

Group 3 -Third sector participant

Some policy, third sector and mother participants perceived an inherent contradiction between simplicity and clarity in the guidance and an understanding that midwifery practice is moving towards a more person-centred model of care. Several midwives noted that a more nuanced communication approach was congruent with a drive towards person-centred antenatal consultations and relationship-building between midwives and pregnant women. Operationalising an absolute abstinence message could lead to reduced trust between midwifes and mothers who wanted to engage with the complexity of the evidence.

\section{Providing reassurance to women who have consumed alcohol}

Some believed that the guidance was intended to help message mediators deliver reassurance to women who had drunk alcohol while encouraging abstinence from then on. However, this reassurance was experienced by midwives and third sector participants as problematic and contradictory. Participants in all groups highlighted the potential to trigger anxiety among women who had drunk alcohol before knowing they were pregnant. Some felt that the guidance encouraged false reassurance in the light of understanding that harm would be more likely in the early weeks of the pregnancy, and if consumption was high:

We have to be open and honest with them, and have to say, 'This is what the guidance says, it's unlikely you've caused any problems, but we don't know'.

Group 2 - Midwife. 


\section{Prevention through surveillance and shaming}

Participants in all groups felt that the guidance increased the likelihood of social surveillance and social shaming but differed as to whether they believed that this was actually intended, and whether it was ethical. For example, some felt that the alcohol industry logo on bottles was an intended and acceptable tool to reinforce social pressure to refrain from drinking alcohol:

[If I saw the logo] I might feel guilty as a drinker, the other is that other people ... I might feel shamed by other people, and those are positive reinforcers.

Group 2 - Midwife

However, many participants saw social surveillance and shaming as inappropriate tools to encourage behaviour change. For some, direct intervention by others into women's decisions was seen an part of a wider cultural norm that accepts interference in what pregnant women's actions

I was like, out having a glass of wine somewhere, at a gig. And someone I kind of knew, but not that well, and she was like, 'Well, you shouldn't be doing that should you?'[She] looked at the bump, looked at the glass of wine. And I was like, well, 'One glass of wine, that's all I've had this week', and she was like, 'Still shouldn't have it, should you.' And I was like, didn't really know her that well, and I was like, 'Cheers for that' $[. .$.$] Soon as you're pregnant... people can say anything.$

Group 4 - New mother

Challenges to guidance coherence

There were three main ways in which the coherence of the guidance on abstention in pregnancy was challenged. These were: (i) the paucity of underpinning evidence base, (ii) the realities of pregnancy (un)planning, and (iii) A wider cultural norm of social drinking.

\section{Lack of evidence of harm}

Participants in all groups tended to feel that the lack of clear evidence around low levels of drinking limited the credibility and coherence of the abstinence message:

It's using terms like 'elusive'... or 'hard to interpret'... It's a strange use of the language around any research finding.

Group 1 - Alcohol policy participant

Several in the policy and third sector groups were concerned that lack of evidence might impact on perceived reliability of public health guidance more broadly:

If you find out that it's not, actually... this very specific statement, 'It's not safe to .... What it if it is? [...] nobody's shown that to be true. How does that impact on public health messages?

Group 3 - Third sector participant

However, despite these limitations, some felt that the risk, in terms of potential damage to the baby, was too great to wait for clearer evidence. Known harm from drinking at higher levels was seen as enough to justify an absolutist approach:

...in the absence of evidence, is it bad for us to say, "It's still No Alcohol, No Risk" [...] which is where the Heads of Midwifery have gone down. They've said: we know that high level drinking causes problems, we've got some sort of bits of evidence that are popping up around low level drinking, and the impact on things like foetal movements, which we know end up in still births, so actually, it's better that we go with 'No alcohol in pregnancy', so that it's out there and it's clear.

Group 1 - Alcohol policy participant

\section{Lived experience of pregnancy planning}


The guidance on abstention during pregnancy planning was problematic in all groups and was considered difficult to operationalise because it assumed a level of pregnancy planning that many participants had not themselves experienced.

Parents, third sector participants and policy makers identified that becoming pregnant could take years, making long-term abstinence unrealistic where it reduced participation in typical social activities:

How far down the line do you have to go? Do you say, 'Don't' drink at all if you're of childbearing age?'That's the whole of your twenties and thirties! That's almost a lifetime. If there's a chance that you might become pregnant, I don't think women think that far in advance.

Group 1 - Alcohol policy participant

Some participants felt that the temporary nature of abstinence in pregnancy was what actually made it more acceptable, while extending this to pre-pregnancy constituted over-reach.

\section{Wider social drinking norms}

Participants in all four groups told personal stories of inadvertent drinking before knowing they were pregnant which, in the context of alcohol being a common part of UK social life, was challenging to avoid.

Parents and third-sector participants also identified that not drinking in social occasions could act as an indicator of pregnancy to other people, which was difficult where women felt not ready to yet disclose this. The anxiety caused by this could be exacerbated by fear of unwanted disclosure to employers and work colleagues. For women trying to get pregnant, not drinking in situations where they would normally do so led to unwanted scrutiny and difficult conversations:

If you're not drinking because you are pregnant then you are in some kind of nice pregnancy club, and then if you're trying... well, then you've got friends around thinking 'So-and-so's not had a drink, I wonder if she's pregnant', and that sort of thing.

Group 4 - New mother

It was widely acknowledged that official guidance was impacted by competition from significant others in mothers' social networks. This included fear of being interpreted as condemning the actions of others who had taken different decisions in response to previous iterations of the guidance:

It's very awkward for you to say, 'Well, actually, your daughter looks lovely, but l'm not going to drink'. And then my sister had a friend whose baby was conceived when she was at the work Christmas party and, you know, as drunk as she'd ever been and I remember my sister saying, 'Well, so-and-so did it and, you know, her baby is fine'.

Group 4 - New mother

Partners' drinking choices were viewed in all groups as significant, with the potential to either help or hinder women's adherence to abstinence guidance. Several mothers described that it was unfair of partners to continue drinking at existing levels when they had to give up. Most participants supported more action to encourage partners to reflect on, and reduce their drinking, both as a statement of support and as an opportunity to reduce their own consumption, maximising the public health benefits of this unique life-stage.

\section{Discussion}

This study adds to understanding of the impact and effectiveness of public health guidance in general and on alcohol and pregnancy guidance more specifically. It's strength is that participants were represented from a range of roles, all having direct experience of the guidance, providing multiple perspectives insights. However, this was a small qualitative study, drawing on a convenience sample of participants meaning a complete range of beliefs and behaviours is not represented. Further research with wider groups of professionals and with mothers from a broader range of socio-economic backgrounds is recommended.

The study highlights contrasting interpretations of current guidance on alcohol in pregnancy, as well as barriers to acceptability and effectiveness. These are considered below in relation to improving guidance acceptability, improving coherence with lived

Page $7 / 12$ 
experience, and improving 'ecological reach'.

Improving acceptability

Acceptability is increasingly acknowledged as an important construct in designing, implementing and evaluating interventions, and a 'necessary but not sufficient' condition for intervention effectiveness ${ }^{11}$. Group discussions highlighted key issues that impacted on the acceptability of the guidelines relating to coherence in the use of the precautionary principle to underpin an abstinence message, with differing views regarding a perceived trade-off between clarity and accuracy.

The guidance prioritises clarity, however where the evidence base is uncertain, a 'clear' message can never be entirely clear. For several participants in this study, particularly policy professionals and midwives, the trade-off between nuance and simplicity felt justified however over-simplification of the evidence may constitute a form of paternalism, which undermines the autonomy of women ${ }^{12}$. Our findings suggest that while some mothers like an abstinence message because it simplifies their own choices, others would prefer more nuanced information than is currently available in the guidance, for example, whether drinking is more harmful in early stages of pregnancy than later. They want to know about the uncertainty in the evidence around risk of drinking at low levels and are interested in the controversy surrounding interpretation. Such interest does not appear to be incompatible with individuals making personal decisions to take a 'better safe than sorry' approach, in line with the conclusions of the guidance, once the evidence has been considered.

There is a need to consider the role of health professionals in mediating the guidance. A communication strategy should relate to existing approaches used by health professionals when talking with mothers. While some health professionals may prefer an unambiguous message, they also recognise the challenge this presents to an ethos of person-centred care. While such tensions are unresolved maternity care staff will, by necessity, decide for themselves which mothers would prefer a simplified approach and which would respond to more complex information, with the potential for this to be an inaccurate judgement. Health professionals lack the time and space to develop individualised communications for pregnant women and would benefit from additional guidance.

Parenting support services with a person-centred focus may feel that lack of nuance is contradictory with their aims and would favour providing opportunity for users to engage with complexity and to consider multiple sources of information. This would involve facilitating service users to access and evaluate information at a level of their own choosing, with access to realistic assessment of the evidence base for those who would like it.

Issues of acceptability also arise in relation to the potential for guidance to cause unintended negative impact. This study is too small to explore such impacts in detail so further work should consider exploring guidance interaction with parental anxiety and impact on mutual support within social networks. We would also suggest further exploration of positive message framing highlighting the gains of abstinence in pregnancy as well as the risks. Although evidence is currently limited, there is some indication that positive messaging can facilitate health prevention behaviours more effectively than negative messages ${ }^{13}$, including intended avoidance of the risk of FASD ${ }^{14}$.

Public health messages in pregnancy occur in a wider culture that tends to take for granted that pregnant women have a duty to minimise all risks to their foetus and are delivered in a moralizing media discourse ${ }^{15}$, where 'everything the pregnant women does and feels (or does not do and does not feel) will impact on the foetus, for better or worse' ${ }^{\prime 16}$. In such a context guidance to abstain from drinking exposes pregnant women who do drink to having their behaviour policed and feeling shamed for their decisions. Guidance should make explicit whether shame is a deliberate mechanism for change (as some of our participants assumed) or an unintended consequence and, where it is the latter, should be reviewed in light of their potential to facilitate unintended harms. Steps to reduce acceptability of shaming and policing should be taken, including through advice to health professionals on more effective tools for managing conversations with women and, potentially, family members.

Acknowledging the lived reality of pregnancy planning

Recommendations to abstain during pre-pregnancy do not accurately reflect current data on the spectrum of pregnancy planning in the UK, with around $16 \%$ of pregnancies unplanned, and a further $30 \%$ of mothers suggesting ambivalence in pregnancy planning ${ }^{17}$. Even when is planned for, the timescale involved is highly unpredictable, making adherence to abstinence guidance problematic. 
Inclusion of pre-pregnancy in current guidance on alcohol consumption both oversimplifies the reality for many women and risks over-reach of the public health role. Communication strategies should carefully consider the risks and benefits of this.

Improving ecological reach

The complex ecological nature of drinking as a part of the social landscape of the UK is not acknowledged in the guidance, which favours individual-behaviour change approaches, with little recognition of the social and interpersonal pressures and influences affecting choice. All stakeholders agreed that women who had made a decision not to drink in pregnancy appreciated support from social network members. Mothers, third sector workers and midwives believed that encouraging partners of expectant mothers to cut down or stop drinking for the duration of the pregnancy would be affirming, would help strengthen the family unit and would reduce the social cost of not drinking among expectant mothers.

The British Medical Association argue that reducing and preventing FASD should be considered within the context of overall reductions in consumption ${ }^{18}$, where population-level change is the desired outcome. Current guidance positions women as decontextualized, individual drinkers with primary responsibility for child health, rather than people living in a society where not drinking is socially unusual and often doesn't receive social support. Drinking decisions are influenced by both cultural and social network norms, with partners of particular importance. It may be helpful to explore approaches to involving partners in behaviour change by highlighting the social influences on drinking and the challenges involved in adherence to current guidance. This may include joint strategies for managing social situations and pregnancy disclosure, as well encouraging reflection on the shared responsibility for child health.

Conclusions: Guidance as an intervention

To improve efficacy of guidance it may be helpful to recognise it as form of intervention in a complex system, and to apply MRC guidance to future development, including establishing and verifying programme theory and incorporating process evaluation to consider social impact of message dissemination and mediation as part of guidance development. The interpretive and coherence challenges, highlighted above, suggest potential benefit in applying intervention theory to understanding acceptability and effectiveness of public health guidance. Discussing development of complex interventions, Craig et al., (2018) ${ }^{19}$ have pointed out that programme theories are often not fully articulated, particularly where the 'intervention' in question is a policy decision. They argue, nonetheless, that those intending to evaluate and improve interventions (including policy decisions) should seek to articulate and develop programme theories, beginning with implicit theory outlined in documents, and in the minds of programme developers and other stakeholders. A key difficulty for understanding, or evaluating, the impact of alcohol in pregnancy guidance is uncertainty as to how the guidance was intended to work and what the impact was intended to be. In other words, description of programme theory for guidance delivery is lacking. The principles underlying the guidance is fuzzy and mechanisms for guidance delivery and the intended role of mediators is largely unspecified. This leads to a vacuum in which stakeholders infer their own understandings about underlying principles and intended change processes. The intended intermediate and long-term outcomes of the abstinence guidance are also unclear - what proportion of drinking in pregnancy, when, among which groups of women, do guidance developers anticipate will be reduced? What is the scale of the intended impact on infant and child health?

In this study, stakeholders inferred a variety of lay programme theories to make sense of the use of the precautionary principle in guidance construction. In some cases, these lay understandings appeared to run counter to the intention of guidance developers; for example, where expectant mothers concluded that the abstinence message was intended for 'more vulnerable women' and not for them. In line with principles of intervention development, underpinning rationales for a decision to adopt a precautionary message (rather than taking an 'informed-choice' approach) could be made transparent. A transparent approach would help inform message delivery and would align with wider principles of honest communication and clear risk presentation ${ }^{20}$.

\section{Abbreviations}

CMO

Chief Medical Officer(s)

FAS

Foetal Alcohol Syndrome 
FASD

Foetal Alcohol Spectrum Disorders

LHB

Local Health Board(s)

\section{Declarations}

Ethics approval and consent to participate: Ethical approval was secured from Cardiff University Research Ethics Committee and written consent was obtained from participants before data collection, including for audio recording.

Consent for publication: Written consent was obtained from participants for inclusion of anonymised quotes within publications.

Availability of data: The scoping review accompanying this work can be accessed at:

https://alcoholchange.org.uk/publication/communicating-public-health-alcohol-guidance-for-expectant-mothers-a-scoping-report-1

Competing interests: Author RB declares no competing interests. Author HT is a trustee of the NCT charity referenced in the research. This is an unpaid position.

Acknowledgements: The authors would like to thank Alcohol Concern Wales for the small grant funding that facilitated this research. We would also like to thank all of our participants for their time and invaluable contribution.

Funding: The work was undertaken with the support of The Centre for the Development and Evaluation of Complex Interventions for Public Health Improvement (DECIPHer), a UKCRC Public Health Research Centre of Excellence. Joint funding (MR/KO232331/1) from the British Heart Foundation, Cancer Research UK, Economic and Social Research Council, Medical Research Council, the Welsh Government and the Wellcome Trust, under the auspices of the UK Clinical Research Collaboration, is gratefully acknowledged.

Author contributions: The study co-authors HT and RB were responsible for all aspects of the study design and completion, as well as publication content and jointly developed all study topic guides, consent and information forms and focus group tasks. The authors jointly convened focus groups and all transcripts were double coded after transcription. The authors and all other relevant parties have consented to publication. All authors have read and approved the manuscript.

\section{References}

1) McAndrew, F., Thompson, J., Fellows, L., Large, A., Speed, M., \& Renfrew, M. J. (2012). Infant feeding survey 2010. Leeds: Health and Social Care Information Centre.

2) O'Keeffe, L. M., Kearney, P. M., McCarthy, F. P., Khashan, A. S., Greene, R. A., North, R. A., . . Dekker, G. A. (2015). Prevalence and predictors of alcohol use during pregnancy: findings from international multicentre cohort studies. BMJ open, 5(7), e006323.

3) Smith, L, Savory, J, Couves, J, Burns, E. (2014) Alcohol consumption during pregnancy: Cross-sectional survey. Midwifery 30 , 1173-1178

4) Patra, J., Bakker, R., Irving, H., Jaddoe, V. W., Malini, S., \& Rehm, J. (2011). Dose-response relationship between alcohol consumption before and during pregnancy and the risks of low birthweight, preterm birth and small for gestational age (SGA)-a systematic review and meta-analyses. BJOG: An International Journal of Obstetrics \& Gynaecology, 118(12), 1411-1421.

5) DOH. (2016). UK Chief Medical Officer's Alcohol Guidelines Review January 2016. London: Department of Health. HM Government. Available at:

https://assets.publishing.service.gov.uk/government/uploads/system/uploads/attachment_data/file/545937/UK_CMOs_report.pdf

6) $\mathrm{DOH}$. (1995). Sensible Drinking. Report of an inter-departmental working group. . London: Department of Health. HM Government

7) Gray, R. \& Henderson, J. (2006) Report to the Department of Health: review of the fetal effects of prenatal alcohol exposure. National Perinatal Epidemiology Unit, University of Oxford. Available at:

https://www.npeu.ox.ac.uk/downloads/files/reports/Alcohol-in-Pregnancy-Report.pdf

Page $10 / 12$ 
8) UK Gov. (2007) Safe. Sensible. Social. - the next steps in the National Alcohol Strategy. HM Government. Available at: https://lx.iriss.org.uk/sites/default/files/resources/Safe\%2C\%20sensible.pdf

9) DoH (2016b) Alcohol Guidelines Review - Report from the Guidelines development group to the UK Chief Medical Officers. Available at:

https://assets.publishing.service.gov.uk/government/uploads/system/uploads/attachment_data/file/545739/GDG_reportJan2016.pdf

10) Braun, V., \& Clarke, V. (2006). Using thematic analysis in psychology. Qualitative research in psychology, 3(2), 77-101.

11) Sekhon, M., Cartwright, M., \& Francis, J. J. (2017). Acceptability of healthcare interventions: an overview of reviews and development of a theoretical framework. BMC health services research, 17(1), 88.

12) Gavaghan, C. (2009). "You can't handle the truth"; medical paternalism and prenatal alcohol use. Journal of medical ethics, 35(5), 300-303.

13) Gallagher, K. M., \& Updegraff, J. A. (2011). Health message framing effects on attitudes, intentions, and behavior: a metaanalytic review. Annals of behavioral medicine, 43(1), 101-116.

14) Yu, N., Ahern, L. A., Connolly-Ahern, C., \& Shen, F. (2010). Communicating the risks of fetal alcohol spectrum disorder: Effects of message framing and exemplification. Health Communication, 25(8), 692-699.

15) Lowe, P., Lee, E., \& Yardley, L. (2010). Under the influence? The construction of foetal alcohol syndrome in UK newspapers. Sociological Research Online, 15(4), 1-10.

16) Lee, E. (2014). Policing pregnancy: The pregnant woman who drinks Parenting culture studies (pp. 129-146): Springer.

17) Wellings, K., Jones, K. G., Mercer, C. H., Tanton, C., Clifton, S., Datta, J., . . Macdowall, W. (2013). The prevalence of unplanned pregnancy and associated factors in Britain: findings from the third National Survey of Sexual Attitudes and Lifestyles (Natsal-3). The Lancet, 382(9907), 1807-1816.

18) British Medical Association (2016) Alcohol and pregnancy: Preventing and managing fetalalcohol spectrum disorders. Available at: https://www.bma.org.uk/collective-voice/policy-and-research/public-and-population-health/alcohol/alcohol-and-pregnancy

19) Craig, P., Di Ruggiero, E., Frohlich, K.L., Mykhalovskiy, E., \& White, M. (2018) on behalf of the Canadian Institutes of Health Research (CIHR) - National Institute for Health Research (NIHR). Taking account of context in population health intervention research: guidance for producers, users and funders of research. Southampton: NIHR Evaluation, Trials and Studies Coordinating Centre; 2018.

20) Qui, W., Rutherford, S., Chu, C., Mao, A., Hou, X. (2016) Risk Communication and public health. Global Journal of Medicine and Public Health. 5(4), 1-11.

\section{Figures}



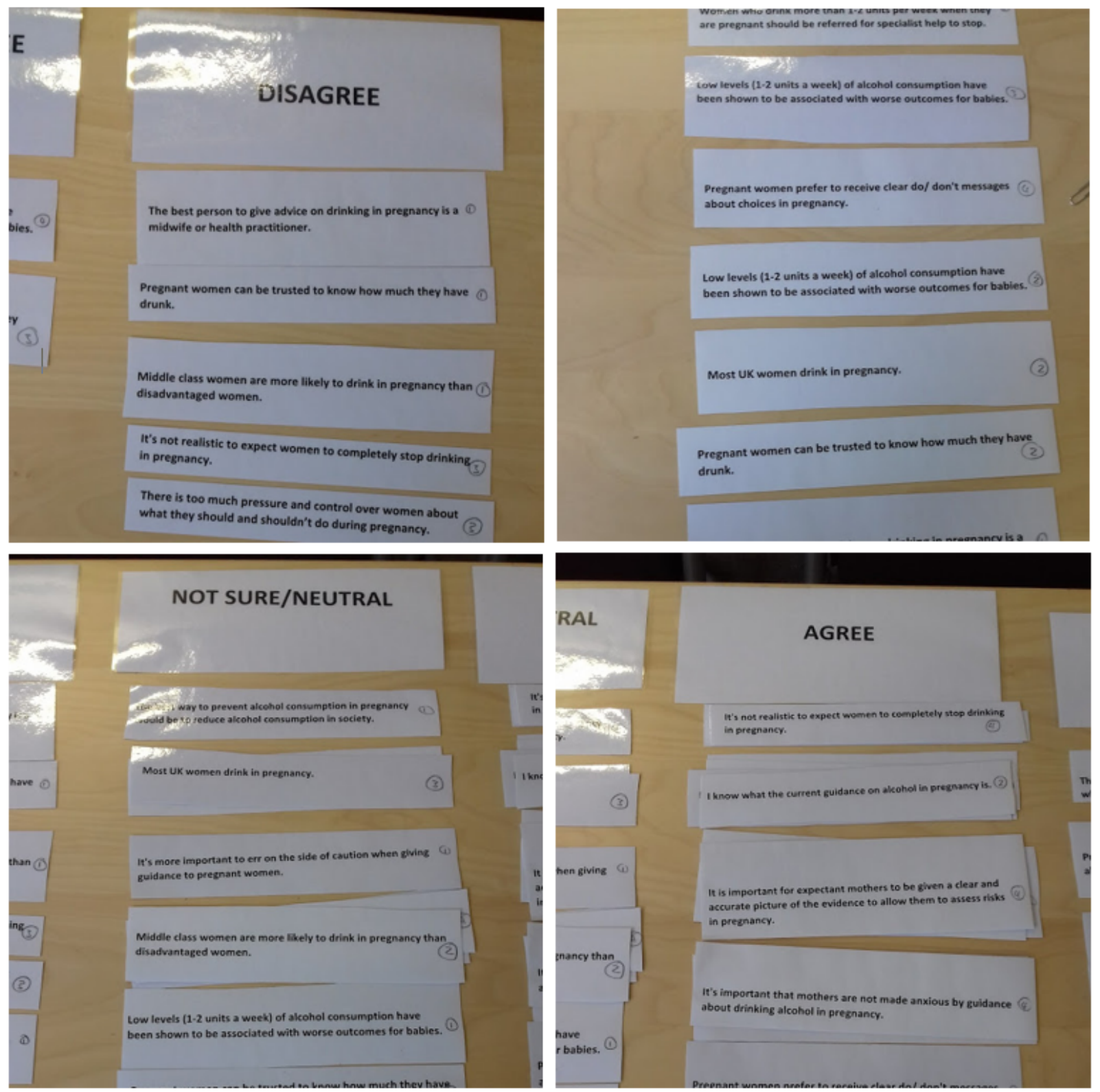

Figure 1

Sample statements sorted by participants as ice-breaker for group discussion

\section{Supplementary Files}

This is a list of supplementary files associated with this preprint. Click to download.

- drafttopicguideAlcoholPregnancygroup1.docx

- ISSMCOREQChecklistalcoholandpregnancypaper.pdf 